\title{
Fibroblast Growth Factor Receptor Family Members as Prognostic Biomarkers in Head and Neck Squamous Cell Carcinoma: A Systematic Review
}

\author{
Norbertus A. Ipenburg ${ }^{1} \cdot \operatorname{Koos~Koole~}^{1} \cdot$ K. Seng Liem ${ }^{1}$. \\ Pauline M. W. van Kempen ${ }^{2} \cdot$ Ron Koole $^{3}$ • Paul J. van Diest ${ }^{1}$. \\ Robert J. J. van Es $^{3}$ • Stefan M. Willems ${ }^{1,4}$
}

Published online: 27 June 2015

(C) The Author(s) 2015. This article is published with open access at Springerlink.com

\begin{abstract}
Background Since head and neck cancer is characterized by poor survival rates, there is a demand for novel therapeutic targets and prognostic biomarkers. An upcoming therapeutic target is the fibroblast growth factor receptor (FGFR) family. However, their prognostic role in head and neck cancer remains unclear.

Objective To systematically review current evidence on the prognostic value of FGFR family members in head and neck squamous cell carcinoma (HNSCC).

Methods A systematic search of PubMed, Embase, and the Cochrane Library was performed for publications up to 14
\end{abstract}

Norbertus A. Ipenburg, Koos Koole and K. Seng Liem contributed equally to this work.

Electronic supplementary material The online version of this article (doi:10.1007/s11523-015-0374-9) contains supplementary material, which is available to authorized users.

Stefan M. Willems

S.M.Willems-4@umcutrecht.nl

Norbertus A. Ipenburg

norbertipenburg@gmail.com

Koos Koole

K.Koole-2@umcutrecht.nl

K. Seng Liem

sengliem@gmail.com

Pauline M. W. van Kempen

P.M.W.vanKempen-2@umcutrecht.nl

Ron Koole

R.Koole@umcutrecht.nl

Paul J. van Diest

P.J.vanDiest@umcutrecht.nl
May 2014. Two reviewers screened all articles and included prognostic studies on the molecular biomarkers FGFR1-5 in any type of HNSCC. Relevant studies were assessed on risk of bias using the Quality in Prognostic Studies (QUIPS) tool. Data on FGFR aberrations and survival outcome were extracted from relevant studies. The prognostic value of FGFR aberrations was compared among studies.

Results The initial search yielded 1568 publications of which 12 fulfilled the inclusion criteria. Four studies reported FGFR1 gene amplification (9.3-17.4\%) and FGFR1 protein overexpression (11.8 \%) in HNSCC. FGFR1 protein expression by cancer-associated fibroblasts correlated with poor
Robert J. J. van Es

R.J.J.vanes@umcutrecht.nl

1 Department of Pathology, University Medical Center Utrecht, PO box 885500,3508 GA Utrecht, The Netherlands

2 Department of Otorhinolaryngology - Head and Neck Surgery, University Medical Center Utrecht, PO box 885500, 3508 GA Utrecht, The Netherlands

3 Department of Oral and Maxillofacial Surgery, University Medical Center Utrecht, PO box 885500, 3508 GA Utrecht, The Netherlands

4 Department of Molecular Carcinogenesis, Netherlands Cancer Institute, PO box 90203, 1006 BE Amsterdam, The Netherlands 
survival outcome in one study $(\mathrm{p}<0.01)$. Eight studies reported high rates of FGFR4 Gly388Arg polymorphisms (32.5$54.2 \%$ ) and FGFR4 protein overexpression (16-35\%), with varying correlations with survival. So far, no studies assessed the prognostic role of FGFR2, FGFR3, or FGFR5 in HNSCC. Limitations Significant risk of bias has been identified among included studies. Therefore, cautious interpretation of the results is recommended.

Conclusion In conclusion, evidence was found for prognostic value of FGFR1 expression in cancer-associated fibroblasts in HNSCC. Prognostic evidence on the other FGFR family members in HNSCC is limited and conflicting. This emphasizes the need for future well-conducted prognostic studies.

\section{Introduction}

With 350,000 deaths each year, head and neck cancer accounts for a significant part of global cancer mortality and is the sixth most common cancer worldwide, affecting more than 650,000 people each year [1]. The most common types of head and neck cancer are laryngeal, oral, and oropharyngeal cancer [2]. Although more than $95 \%$ of these cancers are squamous cell carcinoma, clinical and molecular characteristics of these tumors are heterogeneous [3-5]. Frequently, head and neck squamous cell carcinoma (HNSCC) is detected at an advanced stage implying that the primary tumor has already metastasized to the neck. Advanced stage HNSCC is nowadays treated with varying combinations of radiation therapy, chemotherapy and surgery.

Current chemoradiation therapy regimens cause severe short- and long-term side-effects in more than $80 \%$ of HNSCC patients [6]. Additionally, 5-year relative survival rates have slightly improved over the past three decades, but remain low at $65 \%$. Persisting poor survival rates of HNSCC with current treatment regimens have led to a search for novel therapeutic targets and prognostic biomarkers [6, 7]. The effort to resolve these problems has led to a quest for novel predictive and prognostic biomarkers with the intention to individualize treatment and reserve aggressive therapy for biologically aggressive tumors. As a result, molecular carcinogenesis has become a major focus of cancer research. Previous research endeavors in the pursuit of novel therapeutic targets have identified potential predictive and prognostic molecular biomarkers in HNSCC $[3,8]$. One of them is the fibroblast growth factor receptor (FGFR) family [9].

FGFRs are upcoming promising therapeutic targets and possible prognostic biomarkers in multiple types of cancer, including HNSCC $[9,10]$. The FGFR family comprises five (FGFR1-5) cell membrane-bound tyrosine kinase receptors linked to multiple intracellular downstream signaling pathways. FGFRs regulate tissue homeostasis in normal human tissues [11, 12]. In cancer cells, oncogenic aberrations in FGFR pathway-related genes dysregulate and constitutively activate the FGFR pathway, resulting in particular hallmark capabilities in cancer cells: to sustain proliferative signaling, resist cell death, induce angiogenesis, and activate invasion by cell migration [13-15]. These genomic aberrations include gene fusion, translocation, amplification, and somatic DNA mutations [9]. Because of their major role in cancer cell biology, FGFR family members provide promising opportunities for targeted therapies in a wide spectrum of solid tumors [9, $16,17]$. In addition, previous studies have identified a possible role of FGFRs as prognostic biomarkers, by which they could select patients for adjuvant systemic therapy $[18,19]$. However, the prognostic value of FGFRs in HNSCC remains a subject of debate and has not yet been reviewed in this type of cancer. Therefore, the aim of this study was to systematically review current evidence on the prognostic value of FGFR1-5 in HNSCC and analyze it in a clinically relevant meta-analysis.

\section{Materials and Methods}

\subsection{Eligibility Criteria and Information Sources}

Studies were eligible for inclusion if they were English original articles and addressed the prognostic value of FGFRs in any type of HNSCC (i.e. overall survival, disease-specific survival, disease-free survival, recurrence-free survival, or progression-free survival). FGFR was required to be investigated as a molecular biomarker with laboratory techniques (protein expression, gene amplification, mutation, translation, polymorphism, or mRNA). Original articles were defined as primary research studies with new, unpublished results and written by the researchers who performed the study. Studies were excluded if they investigated fibroblast growth factor (FGF) instead of FGFR, had no prognostic study design, were repetitive studies on same samples, or were non-English. Also, animal studies, case reports, reviews, meta-analysis, and commentaries were excluded. A systematic search of PubMed, Embase, and the Cochrane Library was performed for publications up to 14 May 2014.

\subsection{Search Strategy and Study Selection}

The search terms 'FGFR1-5' and 'head and neck cancer' with all relevant synonyms were used (Online resource 1). Using predefined inclusion and exclusion criteria, two authors (NI and $\mathrm{KL}$ ) independently screened all retrieved records on title and abstract and excluded duplicate titles to select potentially eligible articles. Subsequently, relevant articles were screened on full text, and a further selection of eligible articles was made on relevance of full text. Finally, review articles on this 
topic and references of selected articles were manually screened for titles not identified by the initial search. Selection was based on consensus.

\subsection{Data Collection and Data Items}

Two authors independently (NI and KL) extracted all data of the selected studies using a standardized data extraction form. Discrepancies were resolved by discussion. The following information was extracted from each study: first author's name, year of publication, sample size, head and neck site, treatment, FGFR aberration investigated, type of survival outcome, patient material, laboratory techniques, statistical analysis, cut-off value (if applicable), prevalence of FGFR aberration, correlations with survival outcome, and length of followup.

\subsection{Risk of Bias in Individual Studies}

The methodological quality of the remaining eligible articles was independently assessed by two reviewers (NI and KL), using criteria of the Quality In Prognosis Studies (QUIPS) tool [20]. This tool has been evaluated in 43 groups reviewing prognostic studies and has been identified as a reliable and useful tool for systematic reviewers. Among nine review groups, inter-rater agreement statistic (kappa) varied between 0.56-0.82 [21]. According to the QUIPS tool, risk of bias was scored as low, moderate or high for the following six items; study participation, study attrition, prognostic factor measurement, outcome measurement, study confounding and statistical analysis, and reporting (Online resource 2). Studies scoring low risk of bias on three or more items were considered to be of 'high' methodological quality, while studies scoring high risk of bias on three or more items were considered to be of 'low' methodological quality. All other studies were of moderate quality. The use of proper positive and negative controls for laboratory techniques on FGFR and the use of well-defined scoring criteria for FGFR aberrations were also considered as methodological quality criteria. When there was disagreement on (certain items of) risk of bias of a study, two authors (NI and KL) discussed reasons for disagreement in order to mutually agree on final risk of bias score using the QUIPS tool (Online resource 2).

\subsection{Synthesis of Results}

Because of clinical and methodological heterogeneity of the included studies (study population, type of survival outcome, material, techniques, cut-off values, and applied statistical models), results were not quantitatively pooled. Therefore, a clinically relevant meta-analysis could not be performed. A forest plot was produced using Comprehensive Meta-
Analysis Version 2.2.064 software (Biostat, Englewood, NJ, USA).

\section{Results}

\subsection{Search and Selection}

The initial search yielded 1568 unique articles (Fig. 1). One Chinese article could not be screened because no abstract or full text was available [22]. Therefore, 41 English language, full-text articles were evaluated in more detail, of which 12 original articles met our inclusion criteria. Cross-reference checking revealed no additional relevant publications. Of these 12 included articles, four determined the prognostic value of FGFR1 in HNSCC and eight determined the prognostic value of FGFR 4 by laboratory techniques. Of all 12 studies, Choi et al. had the smallest study population of 24 patients [23]. Seven studies had a study population of mixed HNSCC types, and five studies had a study population of only one type of HNSCC. None of the studies included other types of cancer than HNSCC. In all studies patients were followed in time, but two did not specify the type of survival outcome measured nor the duration of follow-up. All studies accept one analyzed survival outcome using statistical methods such as Log-rank test or Cox proportional hazards model. Furthermore, repetitive studies on similar samples were not found. No articles assessed the prognostic value of FGFR2 or FGFR3 in HNSCC.

\subsection{Quality Assessment}

According to the QUIPS tool, the quality of included studies ranged from high to low. This was mainly because blinding of observers was absent, outcomes were not standardized, and Cox proportional hazard models to control for confounding factors were not performed (Table 1). Three studies did not use proper positive and negative controls for the laboratory techniques [19, 25, 27]. All studies used well-defined criteria for scoring FGFR aberrations. Two studies were of high quality $[18,29]$, four of moderate quality $[19,23,25,30]$, and six studies were of low quality [24, 26-28, 31, 32]. No studies were excluded based on methodological quality.

\subsection{FGFR1 Gene Amplification and FGFR1 Protein Overexpression as Prognostic Biomarkers}

Altogether, four studies on FGFR1 included a total of 993 patients with sample sizes ranging from 61 to 555 samples. Three of them determined FGFR1 gene copy number status and two FGFR1 protein expression (Table 2) [24-27]. Similar fluorescence in situ hybridization (FISH) and immunohistochemical (IHC) techniques were used to determine FGFRI 


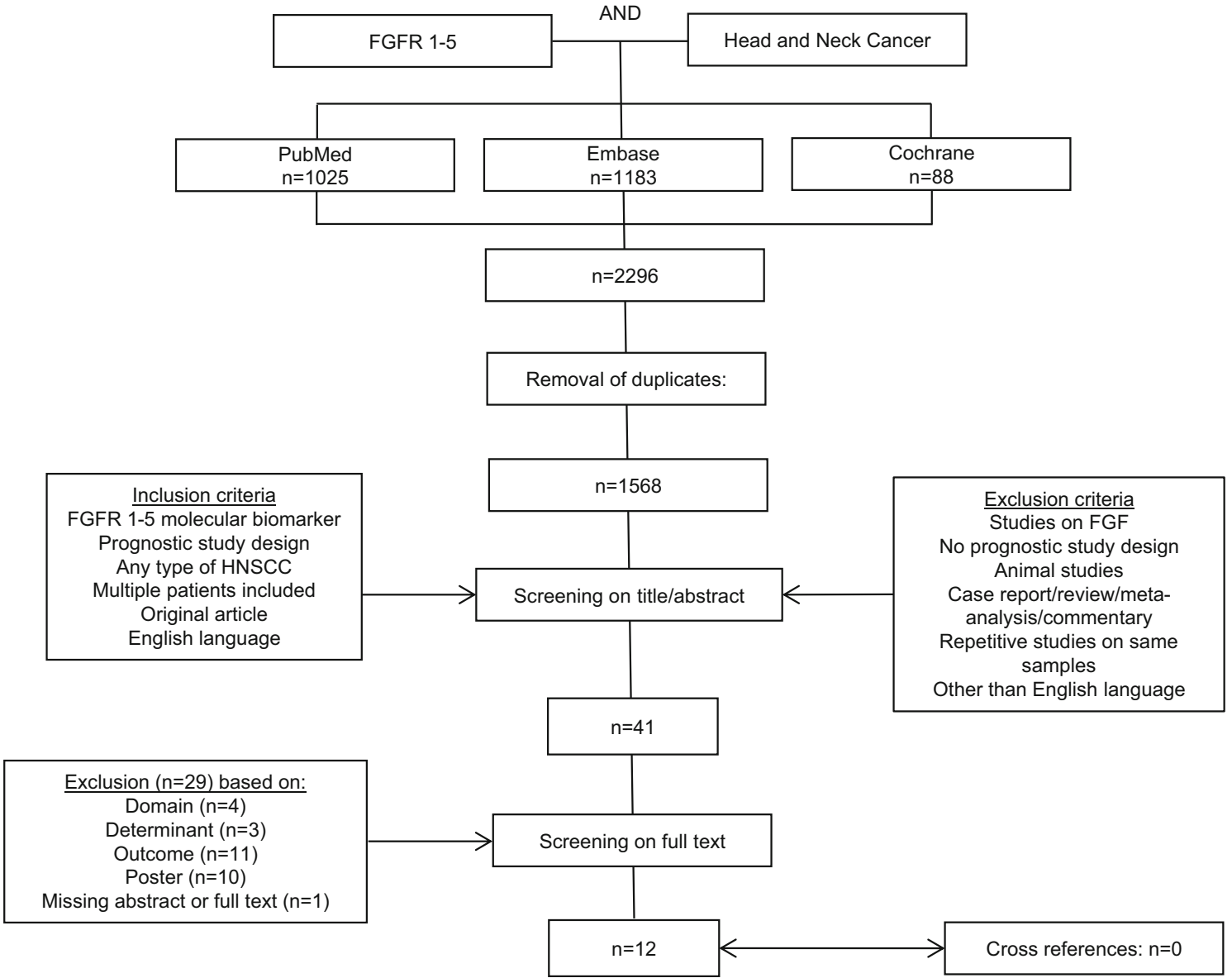

Fig. 1 Flowchart of study selection process (date of search: $14^{\text {th }}$ of May, 2014). FGFR1-5, fibroblast growth factor receptor 1-5; FGF, fibroblast growth factor; HNSCC, head and neck squamous cell carcinoma

Table 1 Quality assessment of studies on the prognostic value of FGFR in HNSCC using the Quality In Prognosis Studies (QUIPS) assessment tool ${ }^{\mathrm{a}}$

\begin{tabular}{|c|c|c|c|c|c|c|c|}
\hline $\begin{array}{l}\text { FGFR } \\
\text { variant }\end{array}$ & Study & $\begin{array}{l}\text { Study } \\
\text { participation }\end{array}$ & $\begin{array}{l}\text { Study } \\
\text { attrition }\end{array}$ & $\begin{array}{l}\text { Prognostic factor } \\
\text { measurement }\end{array}$ & $\begin{array}{l}\text { Outcome } \\
\text { measurement }\end{array}$ & $\begin{array}{l}\text { Study } \\
\text { confounding }\end{array}$ & $\begin{array}{l}\text { Statistical analysis and } \\
\text { reporting }\end{array}$ \\
\hline \multirow[t]{4}{*}{ FGFR1 } & Göke (2013) [24] & ・ & $\bullet$ & ・ & $\bullet$ & $\bullet$ & $\bullet$ \\
\hline & Young (2013) [25] & ' & ' & ' & $\circ$ & $\bullet$ & $\bullet$ \\
\hline & Freier (2007) [26] & $\bullet$ & ' & $\cdot$ & $\bullet$ & $\bullet$ & $\bullet$ \\
\hline & Hase (2006) [27] & ' & ' & $\cdot$ & $\bullet$ & $\bullet$ & $\bullet$ \\
\hline \multirow[t]{8}{*}{ FGFR4 } & Farnebo (2013) [28] & ' & ' & $\circ$ & $\bullet$ & $\bullet$ & $\bullet$ \\
\hline & Dutra (2012) [18] & $\circ$ & ○ & $\cdot$ & $\circ$ & 1 & $\cdot$ \\
\hline & Choi (2012) [23] & ' & ' & $\circ$ & $\circ$ & $\bullet$ & $\bullet$ \\
\hline & Azad (2012) [29] & ' & ○ & $\circ$ & $\circ$ & ○ & $\cdot$ \\
\hline & Tanuma (2010) [30] & ' & ' & $\circ$ & $\bullet$ & ' & $\cdot$ \\
\hline & Ansell (2009) [31] & ' & ' & $\circ$ & $\bullet$ & $\bullet$ & $\bullet$ \\
\hline & $\begin{array}{l}\text { Da Costa Andrade } \\
\text { (2007) [19] }\end{array}$ & ' & ' & $\circ$ & $\bullet$ & ' & ' \\
\hline & Streit (2004) [32] & ' & ' & ' & - & - & - \\
\hline
\end{tabular}

Risk of bias: $\circ=$ low risk; $\bullet=$ moderate risk; $\bullet$ high risk

FGFR, fibroblast growth factor receptor; FGFR1, fibroblast growth factor receptor 1; FGFR4, fibroblast growth factor receptor 4

${ }^{\mathrm{a}}$ The included studies were assessed on items of methodological quality using the QUIPS assessment tool. An elaborate description of each QUIPS item is provided by Hayden et al. [20] and in Online source 2 


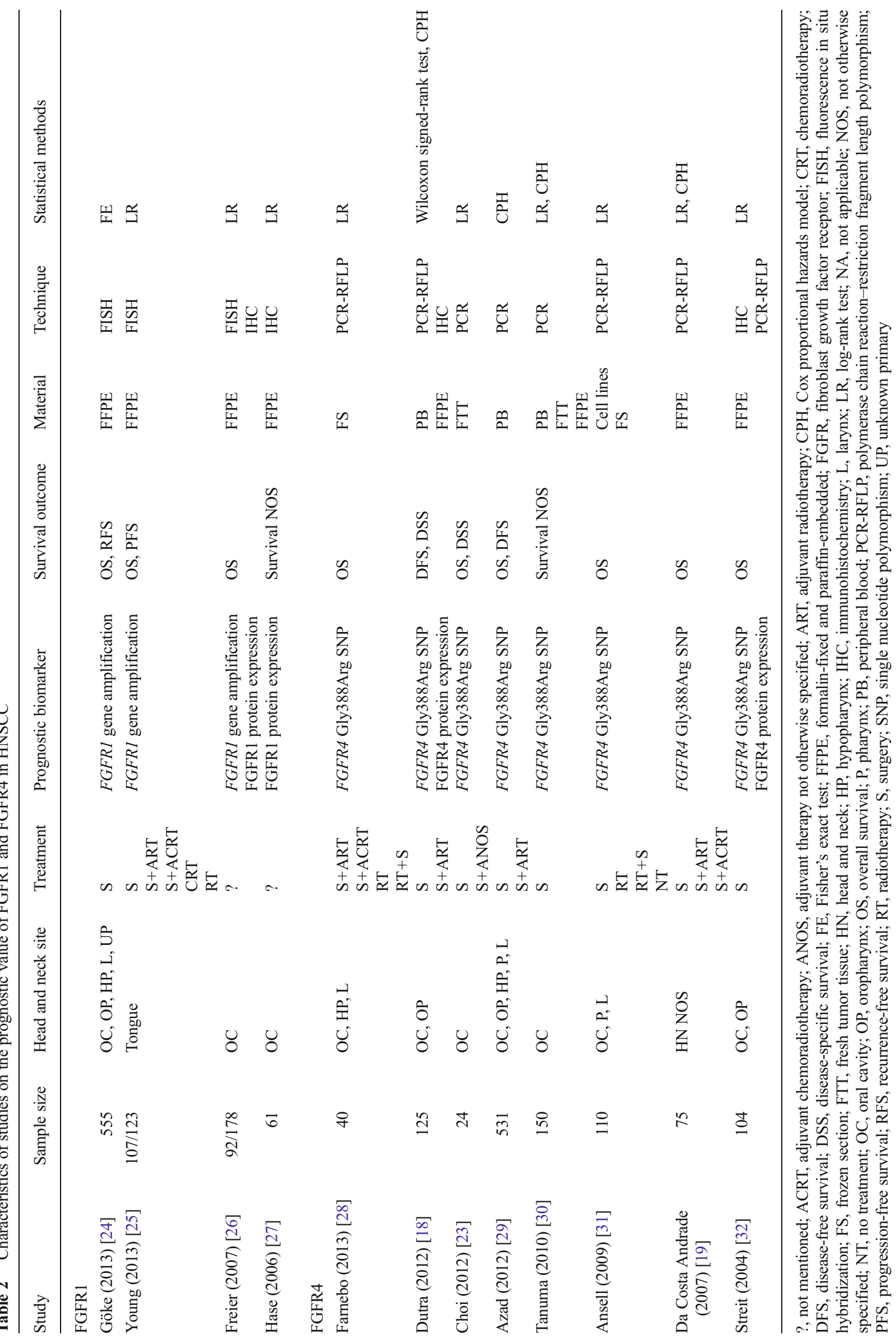


copy number changes and FGFR1 protein expression. Cut-off values for FGFR1 protein overexpression as well as FGFR1 gene amplification varied among these studies (Table 3). In addition, different outcome measures, such as overall survival, progression-free survival, recurrence-free survival, and survival outcome not specified, were used in these studies. None used multivariable Cox proportional hazard models to control for confounding factors.

A high prevalence of FGFR1 gene amplification (9.3$17.4 \%$ ) was found in HNSCC, and a high prevalence FGFR1 protein overexpression was found in oral SCC (11.8 \%; Table 3) [24-27]. FGFR1 gene amplification and FGFR1 protein expression in HNSCC were not significantly correlated to any measure of survival [24-26]. Overall survival and progression-free survival hazard ratios for FGFR1 amplification versus normal copy number pointed in the same direction (overall survival: $\mathrm{HR}=0.94 ; 95 \% \mathrm{CI}=0.37-2.39$; progression-free survival: $\mathrm{HR}=0.79 ; 95 \% \mathrm{CI}=0.24-2.58$; Fig. 2) [25]. However, one study found a significantly worse survival for FGFR1 protein expression in cancer-associated fibroblasts $(p<0.01)$ [27].

\subsection{FGFR4 Gly388Arg Polymorphism and FGFR4 Protein Overexpression as Prognostic Biomarkers}

Eight studies investigated the relationship between FGFR4 Gly388Arg polymorphism and survival in HNSCC; two of them also determined FGFR4 protein expression in relation to survival (Table 2) [18, 19, 23, 28-32]. In total, these studies included 1159 patients with sample sizes ranging from 24 to 531. Study population, head and neck regions, materials and techniques, cut-off values to define protein overexpression, survival outcome measures, and length of follow-up varied considerably among all studies. Regarding statistical analysis, only four studies used a Cox proportional hazards model to control for confounding factors.

Overall, a high prevalence of the FGFR4 Gly388Arg polymorphism (32.5-54.2\%) was found in HNSCC (Table 3). The Arg/Arg genotype occurred in fewer HNSCC cases (5$15.3 \%$ ), whereas wild type (Gly/Gly) occurred more frequently (37.5-67.5\%). Comparing overall survival outcome for the FGFR4 Gly388Arg polymorphism, studies reported longer, equivalent, and even shorter survival rates [19, 23, 28-31]. One study found worse disease-specific survival while another study found no relationship with survival $[18,23]$. Regarding disease-free survival, two studies reported no significant correlation with the FGFR4 Gly388Arg polymorphism $[18,29]$. Overall survival and progression-free survival hazard ratios for the FGFR4 Arg388 allele pointed in opposite directions among different studies (Fig. 2). Because of nonsymmetric lower and upper limit log values, findings on the FGFR4 polymorphism found by Tanuma et al. could not be illustrated in the forest plot [30]. FGFR4 protein overexpression was related to worse overall survival in one study and to worse disease-free survival and diseasespecific survival in another study [18, 32]. Other studies did not investigate the relation of FGFR4 protein expression to survival.

\section{Discussion}

During the past decade, multiple molecular biomarkers which play a role in tumor growth and metastatic spread have been identified. Molecular carcinogenesis has become a major field of cancer research and is driven by the need for novel targetable and prognostic molecular biomarkers. Among these are the FGFR family members [9]. There is increasing evidence for the therapeutic role of FGFRs in HNSCC, but their prognostic role remained unclear.

Herein, we present the first systematic review of currently available evidence on the prognostic value of FGFRs in HNSCC. All included articles were critically appraised using the QUIPS tool items [20]. Studies addressed the prognostic value of FGFR1 gene amplification, FGFR1 protein expression, FGFR4 genotype, and FGFR4 protein expression in $1870 \mathrm{HNSCC}$ cases of various sites, including oral, oropharyngeal, hypopharyngeal, and laryngeal SCC. To date, 12 studies focused on the prognostic value of FGFRs in HNSCC [18, 19, 23-32]. While our extensive search retrieved multiple articles reporting on FGFR2 and FGFR3 in HNSCC, none of these studies assessed their prognostic value [33-39]. Therefore, only FGFR1 and FGFR4 could be assessed as prognostic biomarkers in HNSCC.

Current evidence suggests that FGFR 1 gene amplification and protein overexpression are not prognostic biomarkers of value in HNSCC. However, in one study, FGFR1 protein expression in cancer-associated fibroblasts of HNSCC was a prognostic biomarker which should be validated in a larger cohort [27]. Interestingly, evidence on the prognostic value of the FGFR4 Gly388Arg polymorphism was conflicting [18, 19, 23, 28-32]. This could possibly be explained by differences in duration of follow-up time among studies with positive and negative results. In one study, differences in overall survival only occurred after 24 months of follow-up, while two out of three studies with negative results had a follow-up period of less than three years $[23,31,32]$. This in contrast to positive results in the other four studies with a follow-up time of more than 3 years $[18,19,28,30]$. Remarkably, studies with statistically significant survival results used FFPE tissues, while studies without significant results used DNA from either fresh frozen tumor tissue, peripheral blood, or cell lines [18, 19, 23, 28-32]. Perhaps the 


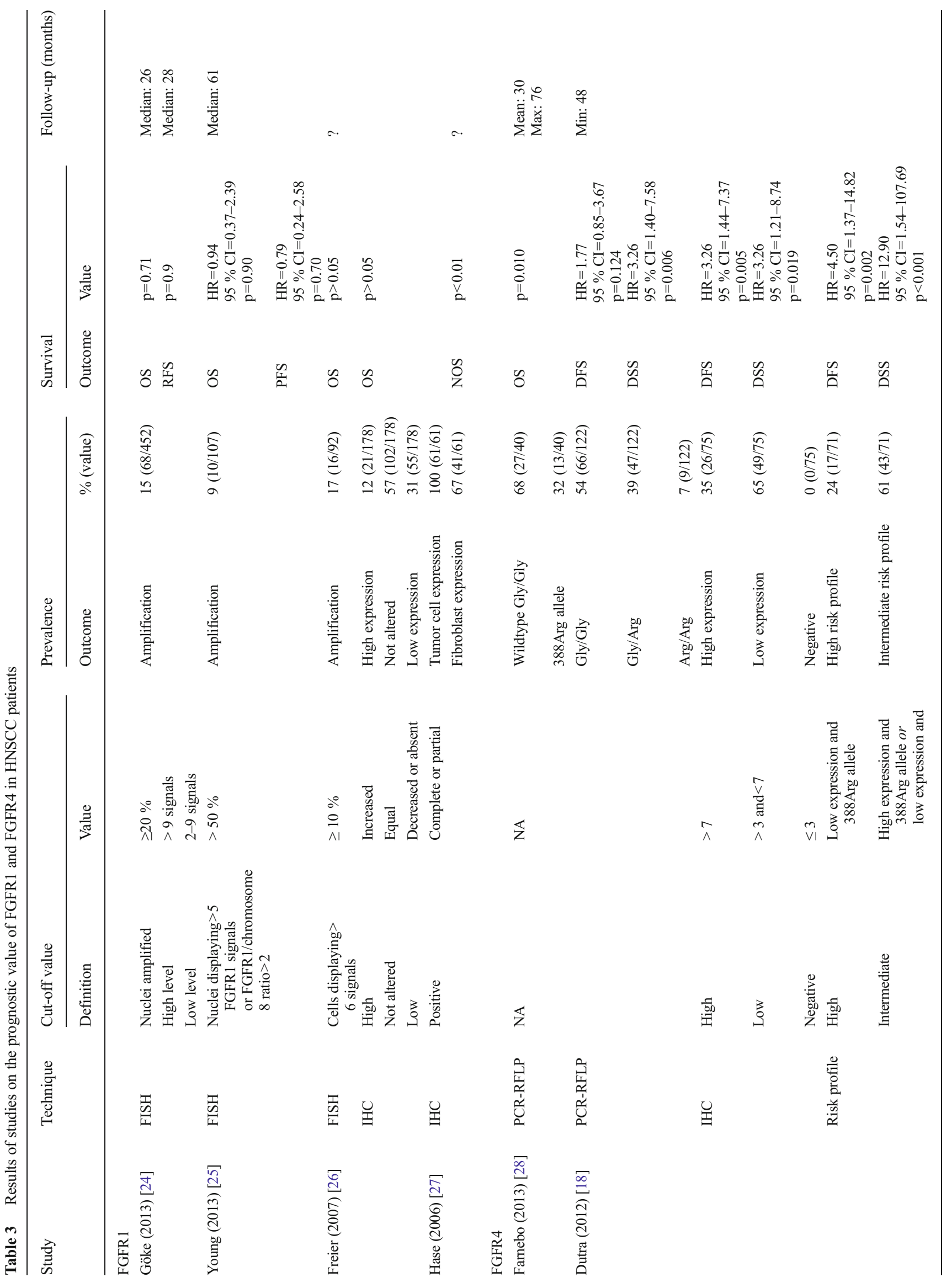




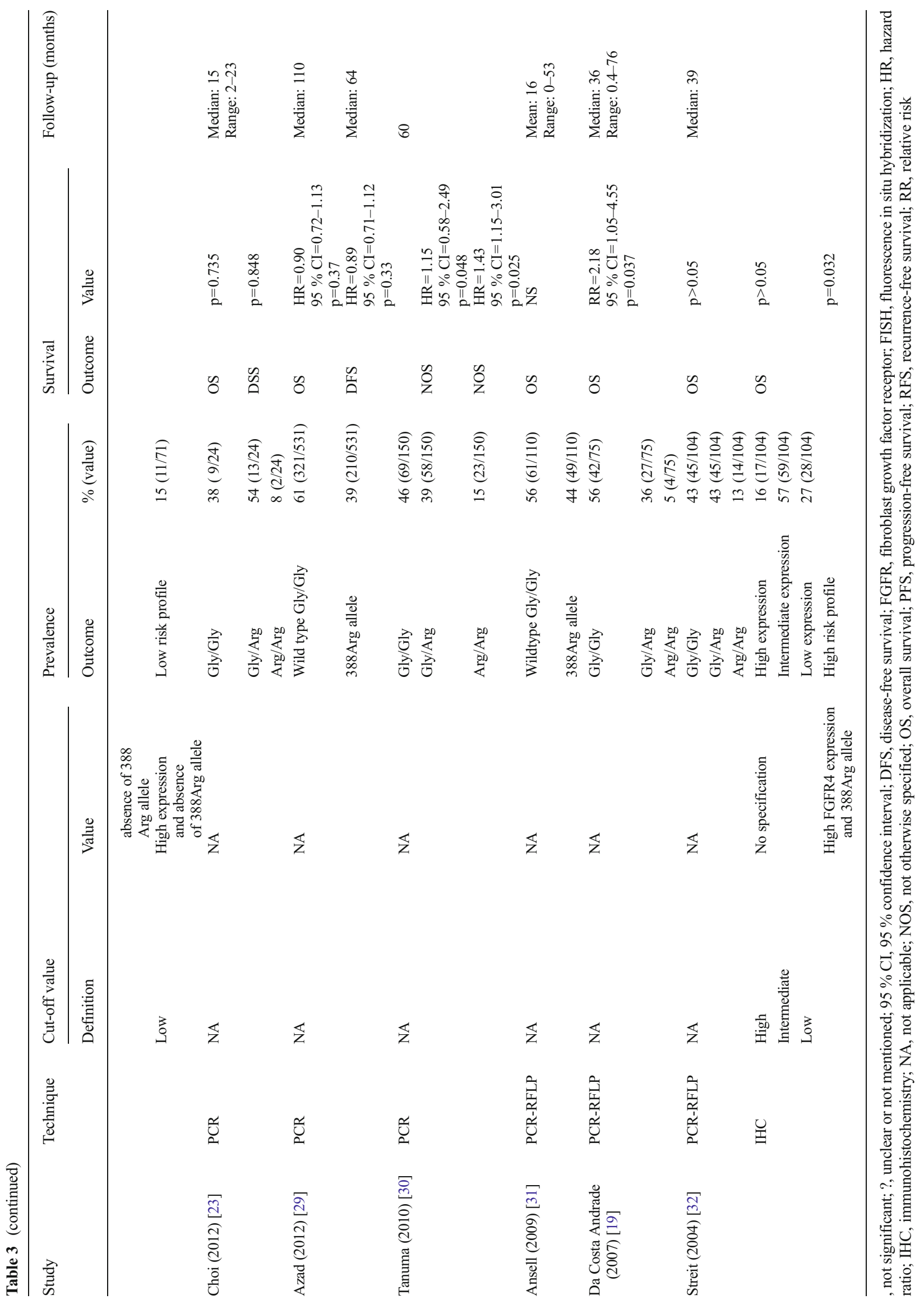




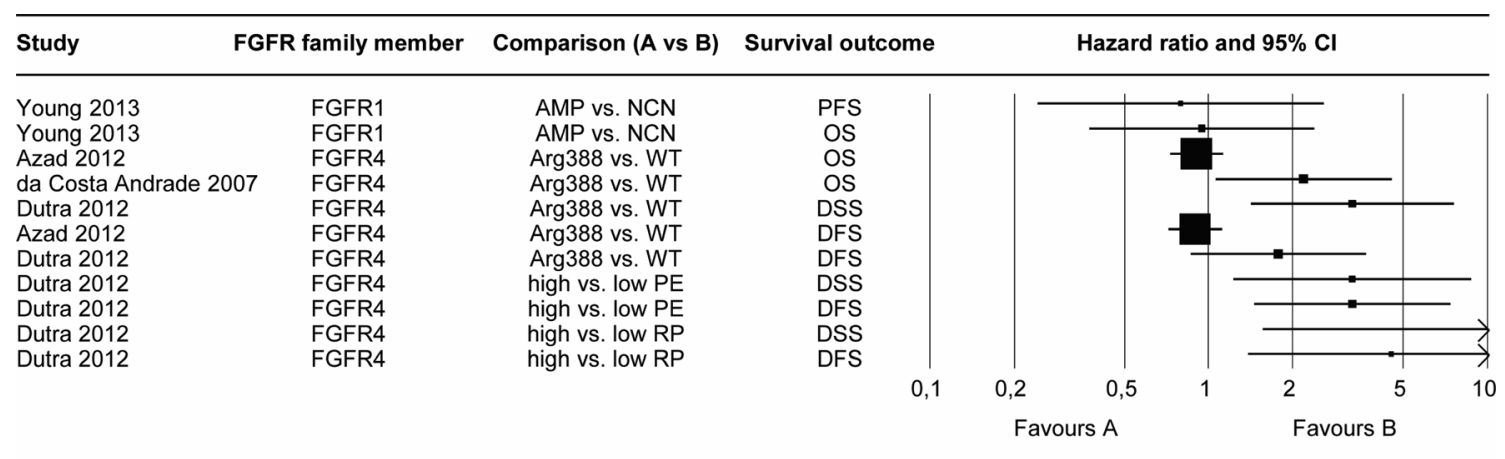

Fig. 2 Forest plot of HNSCC survival grouped by FGFR family member and comparison. AMP, amplification; Arg388, FGFR4 Arg388 allele; CI, confidence interval; DFS, disease-free survival; DSS, disease-specific survival; FGFR, fibroblast growth factor receptor; HR, hazard ratio;

type of study material may influence the PCR techniques used to detect the FGFR4 Gly388Arg polymorphism. Differences in tumor localization of HNSCC might explain the conflicting results on DFS related to the FGFR4 Gly388Arg polymorphism found by Azad et al and Dutra et al $[18,29]$. Azad et al also included hypopharyngeal and laryngeal tumors besides oral and oropharyngeal tumors. HNSCC from different anatomical locations show different clinical and molecular characteristics and are acknowledged as different entities. As such, tumor localization might affect patient outcome. Other differences in study populations or patient treatment could not explain the conflicting results. However, none of the studies were non-randomized controlled trials.

The prognostic value of FGFR has been investigated in numerous other types of cancer. Our findings are in agreement with studies on other types of cancer that also showed conflicting results. For example, studies on FGFR1 gene amplification and FGFR4 Gly388Arg polymorphism in lung SCC [40-46] and breast cancer [47-49] reported conflicting results on overall survival and disease-free survival. Only studies on FGFR 3 mutations in bladder cancer are in agreement with each other; nearly all studies found correlations with better progression-free survival and disease-specific survival [50]. For all other FGFRs in cancer, evidence on their prognostic value is limited or absent and therefore inconclusive, which is similar to HNSCC.

This review focusses on the prognostic value of FGFR aberrations in HNSCC. These prognostic associations do not necessarily predict the response to FGFR-inhibitor therapy as the latter is mainly, yet exclusively, predicted by the underlying aberration itself. For example, previous clinical studies in breast and lung cancer showed that FGFR-inhibitor therapies were only effective in FGFRamplified or FGFR-mutated tumors [51, 52]. The FGFR genes are frequently aberrated in HNSCC, FGFRl is
NCN, normal copy number; NOS, not otherwise specified; OS, overall survival; PE, protein expression; PFS, progression-free survival; RP, risk profile; WT, wild type. Hazard ratios are illustrated as squared boxes and corresponding $95 \%$ confidence intervals are illustrated as error bars

amplified in $10 \%$ of HPV-negative HNSCC and FGFR3 is aberrated in $11 \%$ of HPV-positive HNSCC [53]. As such, HNSCC patients with FGFR-aberrated tumors may benefit from FGFR-inhibitor therapies as these tumors may be sensitive. In addition, targeting FGFR family members has shown to enhance radiotherapy and chemotherapy sensitivity of cancer cells. Radiotherapy resistant cancer cells upregulate FGFR3 protein and chemoradiotherapy resistant cancer cells upregulate FGFR4 protein. Targeting FGFR3 in resistant HNSCC cells restored sensitivity to radiotherapy and targeting FGFR4 restored sensitivity to chemoradiotherapy [54-56].

The probative value of the present review depends on methodological quality of included studies to some extent. All included studies have their specific strengths but the methodological quality is questionable in many. Poor description of study populations and incomplete data in two studies might have introduced selection bias [24, 25]. Six studies had a mean/median follow-up time less than three years [23, 24, 26-28, 31]. Risk of information bias is present in all included studies as none of them provided information on blinding of investigators for the current vital status of patients. Survival rate was only standardized in one study [25]. In all other studies, the definition and assessment of the specific survival outcomes were unclear. Some articles have relatively low sample sizes and did not use multivariable statistical methods, such as multivariable Cox proportional hazard models. Because of risk of bias among included studies, cautious interpretation of results is recommended.

\section{Conclusion}

In conclusion, high quality evidence regarding the prognostic role of FGFR in HNSCC patients is largely lacking. Current 
evidence on FGFR1 in HNSCC is limited to only very few studies. This limited evidence suggests that FGFR1 gene amplification and FGFR1 protein expression are not of value as prognostic biomarkers in HNSCC. However, FGFR1 protein expression in HNSCC-related fibroblasts holds promising prognostic value, but evidence is too limited to draw conclusions yet. The evidence on FGFR4 Gly388Arg polymorphisms in HNSCC is conflicting and inconclusive. FGFR4 protein overexpression may serve as a prognostic biomarker, but evidence is also too limited to draw conclusions yet. Future research should determine the prognostic value of FGFR 1-5 in HNSCC and clarify conflicting results concerning the FGFR4 Gly388Arg polymorphism in HNSC C.

Conflict of interest statement The authors declare that they have no conflict of interest.

Role of the funding source SMW is funded by the Dutch Cancer Society (clinical fellowship: 2011-4964)

The funding source was not involved in the conduct of the research, preparation of the article, study design, writing of the report, or the decision to submit the article for publication.

Open Access This article is distributed under the terms of the Creative Commons Attribution-NonCommercial 4.0 International License (http:// creativecommons.org/licenses/by-nc/4.0/), which permits any noncommercial use, distribution, and reproduction in any medium, provided you give appropriate credit to the original author(s) and the source, provide a link to the Creative Commons license, and indicate if changes were made.

\section{References}

1. Argiris A, Karamouzis MV, Raben D, Ferris RL (2003) Head and neck cancer. Lancet 71:1695-709

2. Pulte D, Brenner H (2010) Changes in survival in head and neck cancers in the late 20th and early 21st century: a period analysis. Oncologist 15:994-1001

3. Seiwert TY, Zuo Z, Keck MK et al (2014) Integrative and comparative genomic analysis of HPV-positive and HPV-negative head and neck squamous cell carcinomas. Clin Cancer Res 21:632-41

4. Van Kempen PM, Noorlag R, Braunius WW et al (2014) Differences in methylation profiles between HPV-positive and HPV-negative oropharynx squamous cell carcinoma: a systematic review. Epigenetics 9:194-203

5. Leemans CR, Braakhuis BJ, Brakenhoff RH (2011) The molecular biology of head and neck cancer. Nat Rev Cancer 11:9-22

6. Conley BA (2006) Treatment of advanced head and neck cancer: what lessons have we learned? J Clin Oncol 24:1023-5

7. Noorlag R, van der Groep P, Leusink FK et al (2014) Nodal metastasis and survival in oral cancer: association with protein expression of SLPI, not with LCN2, TACSTD2, or THBS2. Head Neck. doi: 10.1002/hed.23716

8. Rothenberg SM, Ellisen LW (2012) The molecular pathogenesis of head and neck squamous cell carcinoma. J Clin Invest 122:1951-7
9. Dienstmann R, Rodon J, Prat A et al (2014) Genomic aberrations in the FGFR pathway: opportunities for targeted therapies in solid tumors. Ann Oncol 25:552-63

10. Beenken A, Mohammadi M (2009) The FGF family: biology, pathophysiology and therapy. Nat Rev Drug Discov 8:235-53

11. Powers CJ, McLeskey SW, Wellstein A (2000) Fibroblast growth factors, their receptors and signaling. Endocr Relat Cancer 7:16597

12. Hughes SE (1997) Differential expression of the fibroblast growth factor receptor (FGFR) multigene family in normal human adult tissues. J Histochem Cytochem 45:1005-19

13. Daniele G, Corral J, Molife LR, de Bono JS (2012) FGF receptor inhibitors: role in cancer therapy. Curr Oncol Rep 14:111-9

14. Hanahan D, Weinberg RA (2011) Hallmarks of cancer: the next generation. Cell 144:646-74

15. Haugsten EM, Wiedlocha A, Olsnes S, Wesche J (2010) Roles of fibroblast growth factor receptors in carcinogenesis. Mol Cancer Res 8:1439-52

16. Parker BC, Engels M, Annala M, Zhang W (2014) Emergence of FGFR family gene fusions as therapeutic targets in a wide spectrum of solid tumours. J Pathol 232:4-15

17. Dieci MV, Arnedos M, Andre F, Soria JC (2013) Fibroblast growth factor receptor inhibitors as a cancer treatment: from a biologic rationale to medical perspectives. Cancer Discov 3:264-79

18. Dutra RL, de Carvalho MB, Dos Santos M et al (2012) FGFR4 profile as a prognostic marker in squamous cell carcinoma of the mouth and oropharynx. PLoS One 7, e50747

19. Da Costa Andrade VC, Parise O, Hors CP et al (2007) The fibroblast growth factor receptor 4 (FGFR4) Arg388 allele correlates with survival in head and neck squamous cell carcinoma. Exp Mol Pathol 82:53-7

20. Hayden JA, Côté P, Bombardier C (2006) Evaluation of the quality of prognosis studies in systematic reviews. Ann Intern Med 144: 427-37

21. Hayden JA, van der Windt DA, Cartwright JL et al (2013) Assessing bias in studies of prognostic factors. Ann Intern Med 158:280-6

22. Chen GL, Luo LH, Ding J (2006) Expression of basic fibroblast growth factor and its double receptors in laryngeal squamous cell carcinoma and its significance. Zhonghua Er Bi Yan Hou Tou Jing Wai Ke Za Zhi 41:301-2

23. Choi KY, Rho YS, Kwon KH et al (2013) ECRG1 and FGFR4 single nucleotide polymorphism as predictive factors for nodal metastasis in oral squamous cell carcinoma. Cancer Biomark 12:11524

24. Göke F, Bode M, Franzen A et al (2013) Fibroblast growth factor receptor 1 amplification is a common event in squamous cell carcinoma of the head and neck. Mod Pathol 26:1298-306

25. Young RJ, Lim AM, Angel C et al (2013) Frequency of fibroblast growth factor receptor 1 gene amplification in oral tongue squamous cell carcinomas and associations with clinical features and patient outcome. Oral Oncol 49:576-81

26. Freier K, Schwaenen C, Sticht C et al (2007) Recurrent FGFR1 amplification and high FGFR1 protein expression in oral squamous cell carcinoma (OSCC). Oral Oncol 43:60-6

27. Hase T, Kawashiri S, Tanaka A et al (2006) Correlation of basic fibroblast growth factor expression with the invasion and the prognosis of oral squamous cell carcinoma. J Oral Pathol Med 35:136-9

28. Farnebo L, Tiefenböck K, Ansell A et al (2013) Strong expression of survivin is associated with positive response to radiotherapy and improved overall survival in head and neck squamous cell carcinoma patients. Int J Cancer 133:1994-2003

29. Azad AK, Bairati I, Samson E et al (2012) Validation of genetic sequence variants as prognostic factors in early-stage head and neck squamous cell cancer survival. Clin Cancer Res 18:196-206 
30. Tanuma J, Izumo T, Hirano M et al (2010) FGFR4 polymorphism, TP53 mutation, and their combinations are prognostic factors for oral squamous cell carcinoma. Oncol Rep 23:739-44

31. Ansell A, Farnebo L, Grénman R, Roberg K, Thunell LK (2009) Polymorphism of FGFR4 in cancer development and sensitivity to cisplatin and radiation in head and neck cancer. Oral Oncol 45:23-9

32. Streit S, Bange J, Fichtner A et al (2004) Involvement of the FGFR4 Arg388 allele in head and neck squamous cell carcinoma. Int J Cancer 111:213-7

33. Motahhary P, Baghaie F, Mamishi S et al (2012) Mutational status of FGFR3 in oral squamous cell carcinoma. J Dent (Tehran) 9:7-13

34. Zhang Y, Hiraishi Y, Wang $\mathrm{H}$ et al (2005) Constitutive activating mutation of the FGFR3b in oral squamous cell carcinomas. Int $\mathbf{J}$ Cancer 117:166-8

35. Henson BJ, Gollin SM (2010) Overexpression of KLF13 and FGFR3 in oral cancer cells. Cytogenet Genome Res 128:192-8

36. Sweeny L, Zimmermann TM, Liu Z, Rosenthal EL (2012) Evaluation of tyrosine receptor kinases in the interactions of head and neck squamous cell carcinoma cells and fibroblasts. Oral Oncol 48:1242-9

37. Guancial EA, Werner L, Bellmunt J et al (2014) FGFR3 expression in primary and metastatic urothelial carcinoma of the bladder. Cancer Med 3:835-44

38. Wakulich C, Jackson-Boeters L, Daley TD, Wysocki GP (2002) Immunohistochemical localization of growth factors fibroblast growth factor- 1 and fibroblast growth factor- 2 and receptors fibroblast growth factor receptor- 2 and fibroblast growth factor receptor3 in normal oral epithelium, epithelial dysplasias, and squamous cell carcinoma. Oral Surg Oral Med Oral Pathol Oral Radiol Endod 93:573-9

39. Dellacono FR, Spiro J, Eisma R, Kreutzer D (1997) Expression of basic fibroblast growth factor and its receptors by head and neck squamous carcinoma tumor and vascular endothelial cells. Am J Surg 174:540-4

40. Heist RS, Mino-Kenudson M, Sequist LV et al (2012) FGFR1 amplification in squamous cell carcinoma of the lung. J Thorac Oncol 7:1775-80

41. Craddock KJ, Ludkovski O, Sykes J, Shepherd FA, Tsao M (2013) Prognostic value of fibroblast growth factor receptor 1 gene locus amplification in resected lung squamous cell carcinoma. J Thorac Oncol 8:1371-7

42. Russell PA, Yu Y, Young RJ et al (2014) Prevalence, morphology, and natural history of FGFR1-amplified lung cancer, including squamous cell carcinoma, detected by FISH and SISH. Mod Pathol. doi:10.1038/modpathol.2014.71
43. Kim HR, Kim DJ, Kang DR et al (2013) Fibroblast growth factor receptor 1 gene amplification is associated with poor survival and cigarette smoking dosage in patients with resected squamous cell lung cancer. J Clin Oncol 31:731-7

44. Cihoric N, Savic S, Schneider S et al (2014) Prognostic role of FGFR1 amplification in early-stage non-small cell lung cancer. Br J Cancer 110:2914-22

45. Seo AN, Jin Y, Lee HJ et al (2014) FGFR1 amplification is associated with poor prognosis and smoking in non-small-cell lung cancer. Virchows Arch. doi:10.1007/s00428-014-1634-2

46. Bange J, Prechtl D, Cheburkin Y et al (2002) Cancer progression and tumor cell motility are associated with the FGFR4 Arg 388 allele. Cancer Res 62:840-7

47. Thussbas C, Nahrig J, Streit S et al (2006) FGFR4 Arg388 allele is associated with resistance to adjuvant therapy in primary breast cancer. J Clin Oncol 24:3747-55

48. Jézéquel P, Campion L, Joalland MP et al (2004) G388R mutation of the FGFR4 gene is not relevant to breast cancer prognosis. Br J Cancer 90:189-93

49. Spinola M, Leoni VP, Tanuma J et al (2005) FGFR4 Gly388Arg polymorphism and prognosis of breast and colorectal cancer. Oncol Rep 14:415-9

50. Liu X, Zhang W, Geng D et al (2014) Clinical significance of fibroblast growth factor receptor-3 mutations in bladder cancer: a systematic review and meta-analysis. Genet Mol Res 13:1109-20

51. Hammerman PS, Hayes DN, Grandis JR (2015) Therapeutic insights from genomic studies of head and neck squamous cell carcinomas. Cancer Discov 5:239-44

52. André F, Bachelot $\mathrm{T}$, Campone $\mathrm{M}$ et al (2013) Targeting FGFR with dovitinib (TKI258): preclinical and clinical data in breast cancer. Clin Cancer Res 19:3693-702

53. The Cancer Genome Atlas Network (2015) Comprehensive genomic characterization of head and neck squamous cell carcinomas. Nature 517:576-82

54. Uzawa K, Ishigami $\mathrm{T}$, Fushimi $\mathrm{K}$ et al (2011) Targeting fibroblast growth factor receptor 3 enhances radiosensitivity in human squamous cancer cells. Oncogene 30:4447-52

55. Roidl A, Berger H, Kumar S et al (2009) Resistance to chemotherapy is associated with fibroblast growth factor receptor 4 up-regulation. Clin Cancer Res 15:2058-66

56. Turkington RC, Longley DB, Allen WL et al (2014) Fibroblast growth factor receptor 4 (FGFR4): a targetable regulator of drug resistance in colorectal cancer. Cell Death Dis 5, e1046 